\title{
Erratum to: Analytic derivation of the Cox-Merz rule using the MLD "toy" model for polydisperse linear polymers
}

\author{
Springer Production
}

Published online: 21 September 2011

(C) Springer-Verlag 2011

\section{Erratum to: Rheol Acta}

\section{DOI 10.1007/s00397-011-0550-5}

The given affiliation "Center for the Molecular Study of Condensed Soft Matter, Illinois Institute of Technology, Chicago, IL 60616, USA" was illegitimately stated by the author of the original article D. W. Mead. The author may be contacted by the email address given in the original article. 